\title{
Simplified Bernoulli Formula to Diagnose Ischemia-Causing Stenosis at Coronary CT Angiography: Comparison with SPECT
}

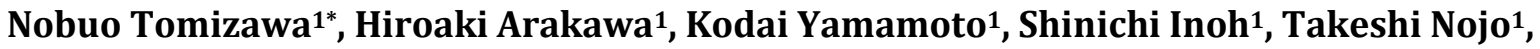 \\ Sunao Nakamura ${ }^{2}$
}

${ }^{1}$ Department of Radiology, New Tokyo Hospital, Tokyo

${ }^{2}$ Department of Cardiology, New Tokyo Hospital, Tokyo

Email: ^tomizawa-tky@umin.ac.jp

How to cite this paper: Tomizawa, N., Arakawa, H., Yamamoto, K., Inoh, S., Nojo, T. and Nakamura, S. (2019) Simplified Bernoulli Formula to Diagnose Ischemia-Causing Stenosis at Coronary CT Angiography: Comparison with SPECT. Advances in Computed Tomography, 8, 11-23.

https://doi.org/10.4236/act.2019.82002

Received: March 8, 2019

Accepted: May 5, 2019

Published: May 8, 2019

Copyright (c) 2019 by author(s) and Scientific Research Publishing Inc. This work is licensed under the Creative Commons Attribution International License (CC BY 4.0).

http://creativecommons.org/licenses/by/4.0/

\section{(c) (i) Open Access}

\begin{abstract}
Purpose: To compare the diagnostic performance of estimated energy loss (EEL) calculated using a simplified Bernoulli formula at coronary computed tomography (CT) and single photon emission computed tomography (SPECT) to diagnose ischemia-causing stenosis by invasive fractional flow reserve (FFR). Methods: We retrospectively included 43 patients who underwent coronary CT, SPECT, and FFR measurement by catheter within 3 months. When an intermediate stenosis $(40 \%-70 \%)$ was present at CT, EEL was calculated using the following parameters: lesion length, diameter stenosis, minimal lumen area, and the myocardial volume. An EEL $>1.17$ or diameter stenosis $>70 \%$ was determined ischemic. Stress-induced ischemia by SPECT was determined when a perfusion defect at stress was accompanied with a fill-in at rest. An FFR $\leq 0.80$ or diameter stenosis $>70 \%$ was determined as ischemic by catheter. Results: A total of 26 vessels were determined as ischemic by catheter exam. The per-vessel sensitivity and specificity of EEL and SPECT were $81 \%$ vs $42 \%$ and $92 \%$ vs $91 \%$, respectively. The accuracy of EEL to diagnose stenosis causing ischemia was significantly higher than SPECT ( $90 \%$ vs $81 \%, p=0.04$ ). The area under the curve of the receiver operating characteristics curve was also significantly higher for EEL than SPECT (0.86 vs $0.67, p<0.005)$. Conclusions: EEL showed higher accuracy than SPECT to diagnose ischemia-causing stenosis by improving the sensitivity.
\end{abstract}

\section{Keywords}

Coronary Computed Tomography Angiography, Estimated Energy Loss, Fractional Flow Reserve 


\section{Introduction}

Growing evidence has shown that coronary artery disease treated based on functional ischemia improves the patient outcome than anatomically-guided methods [1]. Hence, functional assessment of coronary stenosis is important. Diagnosis of coronary ischemia by single photon emission computed tomography (SPECT) has abundant evidence, but additional cost and stress agent is necessary to perform this method. Fractional flow reserve (FFR) is another method to diagnose ischemia during catheter exam. Recently, computing the FFR using coronary CT angiography data is available [2]. Although FFR derived from CT could correctly predict ischemia by invasive FFR with an accuracy of $80 \%$ [2], coronary CT data needs to be transferred to an external supercomputer and turn-around time is necessary.

Simplified Bernoulli formula is frequently used at echocardiography to estimate the pressure loss across a valvular stenosis. This method could also be used to calculate estimated energy loss (EEL), which is an index to estimate the pressure loss across a coronary stenosis. A previous study showed that EEL could correctly predict ischemia assessed by FFR with an accuracy of $83 \%$ [3]. However, the diagnostic performance of EEL has not been compared with SPECT which is the current clinical standard to diagnose myocardial ischemia. Thus, the purpose of this study was to compare the diagnostic performance of EEL and SPECT to predict ischemia-causing stenosis by invasive FFR.

\section{Materials and Methods}

\subsection{Patients}

This retrospective study was approved by the local ethics committee, and the requirement for informed consent to participate this study was waived. The records of 55 patients who underwent FFR evaluation during coronary angiography and SPECT myocardial perfusion imaging because coronary artery disease was suspected by CT angiography from April 2014 to November 2018 were retrospectively examined. The following patients were excluded: interval between SPECT and CT $>3$ months $(\mathrm{n}=5)$, interval between catheter exam and CT $>3$ months $(\mathrm{n}=4)$, severe motion artifact at coronary CT angiography $(\mathrm{n}=$ $1)$, FFR only performed in a vessel with suspected in-stent restenosis $(n=1)$, and caffeine intake before coronary angiography $(n=1)$. Thus, the final study group included 43 patients. Of the assessable 129 vessels, the following two vessels were excluded: FFR not performed despite intermediate stenosis and hypoplastic right coronary artery due to left dominance. The pretest probability of coronary artery disease was estimated by a previously described method [4].

\subsection{Coronary CT Angiography Acquisition}

All patients underwent CT angiography with a 64-row CT (Brilliance 64; Philips, Eindhoven, Netherlands, or Somatom Definition AS+; Siemens Healthineers, Forchheim, Germany). 
Patients with heart rate $>65$ beats/min at the outpatient department were told to take an oral $\beta$-blocker (20 mg of metoprolol) $1 \mathrm{~h}$ prior to CT angiography. If the heart rate was over 65 beats/min on site, a maximum dose of $12.5 \mathrm{mg}$ of landiolol (Corebeta; Ono Pharmaceutical, Tokyo, Japan) was given intravenously [5]. All patients received $0.3 \mathrm{mg}$ sublingual nitroglycerin (Nitropen; Nippon Kayaku, Tokyo, Japan) before imaging.

The scanning parameters for Brilliance 64 were as follows. Calcium scoring scan was performed by a prospective electrocardiogram-gated axial scan with $120 \mathrm{kVp}, 196 \mathrm{~mA}$ and $2.5 \mathrm{~mm}$ collimation. Images were acquired at $40 \%$ or $70 \%$ of the R-R interval depending on the heart rate and were reconstructed with a slice thickness of $2.5 \mathrm{~mm}$ and increment of $2.5 \mathrm{~mm}$. Coronary CT angiography was performed by electrocardiogram-gated helical scan with dose modulation technique and the following parameters: Detector configuration, $64 \times 0.625 \mathrm{~mm}$; tube potential, $120 \mathrm{kVp}$; tube current-time product, 800 - $1050 \mathrm{mAs}$, depending on the body weight; gantry rotation time, $420 \mathrm{~ms}$; and helical pitch, 0.2 . The reconstructed slice thickness was $0.67 \mathrm{~mm}$, and the increment was $0.33 \mathrm{~mm}$. Images were reconstructed using a cardiac sharp kernel. Iterative reconstruction technique was not available with this scanner.

The scanning parameters for Definition AS+ were as follows. Calcium scoring scan was performed by a prospective electrocardiogram-gated axial scan with $120 \mathrm{kVp}$ and a reference mAs of $40 \mathrm{mAs}$. Coronary CT angiography was performed by prospective electrocardiogram-gated axial scan. The tube potential and the reference mAs were set as $120 \mathrm{kVp}$ and $250 \mathrm{mAs}$. The acquisition window initiated at $60 \%-75 \%$ of the R-R interval when the heart rate was below 65 beats/min, otherwise initiated at 30\% - 75\%. The reconstructed slice thickness was $0.75 \mathrm{~mm}$ and the increment was $0.4 \mathrm{~mm}$ using a cardiac kernel (I36f) with sinogram-affirmed iterative reconstruction strength 2 .

For processing, images were transferred to a workstation (Synapse Vincent Ver 5.1; Fujifilm Medical, Tokyo, Japan).

\subsection{Coronary CT Angiography Analysis}

Calcified lesions with a minimum area of three pixels and a minimum CT number of $130 \mathrm{HU}$ were scored by using the algorithm developed by Agatston et al. [6]. Lesion scores from the left main, left anterior descending, left circumflex, and right coronary arteries were summed to determine a total calcium score. Calcium score was not calculated in patients with stents.

When an intermediate stenosis (40\% - 70\%) was present, EEL was calculated by a previously described method [3]. Briefly, the energy loss across a coronary stenosis was calculated using the following parameters: Lesion length (LL), diameter stenosis (DS), minimal lumen area (MLA), and the myocardial volume of the stenosis-related territory. Lesion length, diameter stenosis, and minimal lumen area were automatically obtained using the stenosis analysis software of the workstation (Figure 1(a)). The left ventricular volume of the stenosis-related territory was estimated using the Voronoi method (Figure 1(b)). The coronary 


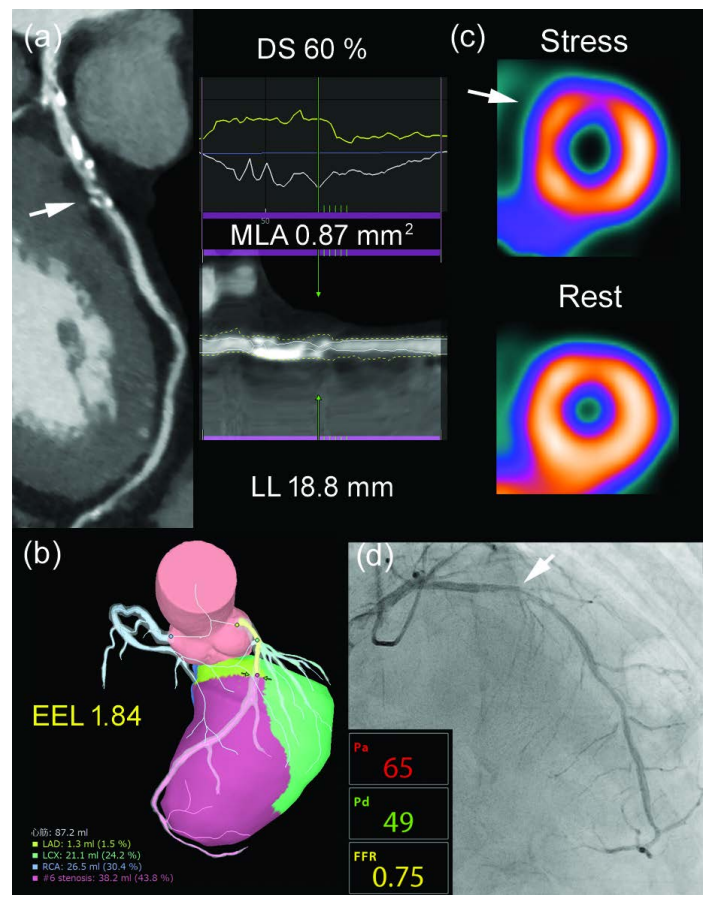

Figure 1. Curved planar reconstruction image and stenosis analysis performed by the software of an 81-year-old male with chest discomfort on effort (a); Diffuse calcification was present at the proximal left anterior descending (a, arrow) with $60 \%$ stenosis, MLA of $0.87 \mathrm{~mm}^{2}$, and LL of $18.8 \mathrm{~mm}$. The myocardial volume of the stenosis-related territory was $38.2 \mathrm{ml}$ (b); EEL was calculated as 1.84, above the threshold for ischemia $(>1.17)$. SPECT also showed stress-induced ischemia at the anteroseptal wall ((c), arrow). Angiography of the left anterior descending showed an intermediate stenosis ((d), arrow) and FFR value of 0.75 was positive for ischemia $(\leq 0.80)$ (d). DS, diameter stenosis; EEL, estimated energy loss; FFR, fractional flow reserve; LL, lesion length; MLA, minimal lumen area; SPECT, single photon emission computed tomography.

flow at maximal hyperemia was estimated at $4.0 \mathrm{ml} \cdot \mathrm{min}^{-1} \cdot \mathrm{g}^{-1}$. Using a simplified Bernoulli formula, pressure loss across a stenosis can be estimated as follows [7].

$$
\Delta P=Q \frac{8 \pi \mu L L}{M L A^{2}}+Q^{2} \frac{\rho}{2} \frac{\left(1-(1-D S / 100)^{2}\right)^{2}}{M L A^{2}}
$$

where $\Delta P$ is the pressure drop, $Q$ is the blood flow, $\mu$ is the blood viscosity $(0.004$ $\left.\mathrm{kg} \cdot \mathrm{m}^{-1} \cdot \mathrm{s}^{-1}\right)$ and $\rho$ is the blood density $\left(1000 \mathrm{~kg} \cdot \mathrm{m}^{-3}\right)$. The first term accounts energy loss due to viscous friction between laminar layers of fluid and the second term reflects energy loss when normal arterial flow is transformed first to high-velocity flow in the stenosis and then to the turbulent nonlaminar distal flow eddies at the exit from the stenosis. We defined EEL as log $\triangle P$ and determined EEL $>1.17$ as ischemic [3].

\subsection{FFR Evaluation}

FFR was performed using a pressure wire (PressureWire Cetrus; St Jude Medical Systems, St. Paul, MN) when the stenosis was between $40 \%-70 \%$ at invasive coronary angiography. The pressure wire was positioned distal to the stenosis of 
interest under intravenous infusion of adenosine triphosphate (Adetphos; Kowa Company, Tokyo, Japan) at $140 \mu \mathrm{g} \cdot \mathrm{kg}^{-1} \cdot \mathrm{min}^{-1}$ for $3 \mathrm{~min}$. FFR was derived as the ratio of the mean coronary pressure distal to the stenosis over the mean aortic pressure at maximal hyperemia. An FFR value of $\leq 0.80$ was determined as hemodynamically significant. The cardiologists performing angiography had total access to the coronary CT data. However, they were not aware of EEL because this value was calculated for research use and not mentioned in the radiology report.

\subsection{SPECT Myocardial Perfusion Imaging Acquisition and Analysis}

SPECT was performed using ${ }^{99 \mathrm{~m}} \mathrm{Tc}$-tetrofosmin (Myoview; Nihon Medi-Physics, Tokyo, Japan) with $296 \mathrm{MBq}$ used for stress and $740 \mathrm{MBq}$ used for rest studies. Pharmacologic stress was performed using intravenous infusion of adenosine (Adenoscan; Daiichi Sankyo Healthcare, Tokyo, Japan) at $120 \mu \mathrm{g} \cdot \mathrm{kg}^{-1} \cdot \mathrm{min}^{-1}$ for 6 min. Electrocardiographic gating on a dual-headed SPECT system was performed with 8 frames per cardiac cycle (Infinia Hawkeye 4; GE Healthcare, Milwaukee, WI). Two independent observers visually interpreted the images and joint consensus reading was performed when discrepancy was present. Stress-induced ischemia was determined when a perfusion defect at stress was accompanied with a fill-in at rest (Figure 1(c)).

\subsection{Statistical Analysis}

Continuous variables were shown as mean \pm standard deviation and categorical variables as number (\%) unless otherwise described. The Student $t$-test was used to compare continuous variables. The Chi square $\left(\chi^{2}\right)$ test and the Fisher exact test were used to compare categorical and skewed variables. Interobserver agreement for diagnosis of perfusion defect at SPECT was calculated using the Cohen $\kappa$ statistic [8], which was interpreted as poor $(\kappa<0.20)$, fair $(\kappa=0.21-$ $0.40)$, moderate $(\kappa=0.41-0.60)$, good $(\kappa=0.61-0.80)$, very good $(\kappa=0.81$ $0.90)$, or excellent $(\kappa \geq 0.91)$. The McNemar test was used to assess the difference in the diagnostic accuracy. Receiver operating characteristics (ROC) curve analysis was performed to compare the diagnostic performance of EEL and SPECT to predict ischemia-causing stenosis by FFR. The difference in area under the curve (AUC) was assessed by the DeLong method [9] using R (The R Foundation for Statistical Computing). The remaining statistical analyses were performed using JMP software (version 12.2.0; SAS, Cary, NC). A $p$-value $<0.05$ was deemed to indicate significance.

\section{Results}

\subsection{Patient Demographics}

The majority of patients (74\%) were male and the coronary risk was moderate (Table 1). The median (interquartile range) calcium score was high with a value 
Table 1. Patient demographics.

\begin{tabular}{|c|c|}
\hline & \\
\hline Number of patients & 43 \\
\hline Male & $32(74)$ \\
\hline Age $(y)$ & $73.6 \pm 6.6$ \\
\hline Body weight (kg) & $60.6 \pm 11.1$ \\
\hline Body mass index $\left(\mathrm{kg} / \mathrm{m}^{2}\right)$ & $23.1 \pm 2.6$ \\
\hline \multicolumn{2}{|l|}{ Coronary risk factors } \\
\hline Diabetes mellitus & $12(28)$ \\
\hline Hypertension & $36(84)$ \\
\hline Dyslipidemia & $29(67)$ \\
\hline Smoking & $31(72)$ \\
\hline Family history & $11(26)$ \\
\hline Risk score ${ }^{*}$ & $12.5 \pm 2.8$ \\
\hline Post PCI & $3(7)$ \\
\hline Calcium score $\dagger$ & $468.7(142.5-848.7)$ \\
\hline \multicolumn{2}{|l|}{ Disease severity $\ddagger$} \\
\hline 1 vessel disease & $11(26)$ \\
\hline 2 vessel disease & $6(14)$ \\
\hline 3 vessel disease & $1(2)$ \\
\hline \multicolumn{2}{|l|}{ Disease location } \\
\hline Right coronary artery & $3(7)$ \\
\hline Left anterior descending artery & $14(33)$ \\
\hline Left circumflex artery & $9(21)$ \\
\hline
\end{tabular}

Numbers are reported as average \pm standard deviation or $N(\%)$, unless otherwise described. ${ }^{\star}$ The risk score was determined by the criteria by Tomizawa et al. [4]. Median (interquartile range). An FFR value $\leq 0.80$ or diameter stenosis $\geq 70 \%$ was determined hemodynamically significant. FFR, fractional flow reserve; PCI, percutaneous coronary intervention.

of 468.7 (142.5 - 848.7). Eighteen patients (42\%) had at least one vessel with ischemia proven by FFR $(\leq 0.80)$ or severe stenosis (diameter stenosis $>70 \%)$. FFR was measured in 49 vessels and the average value was $0.83 \pm 0.10$. The ischemic vessel was most frequent at the left anterior descending (14 vessels $(33 \%)$ ), followed by the left circumflex (9 vessels (21\%)), and the right coronary artery (3 vessels $(7 \%))$.

\subsection{Per-Vessel Analysis}

CT angiography showed severe stenosis (diameter stenosis $>70 \%$ ) in 8 vessels and the calculated EEL was significant (>1.17) in 21 vessels. Hence, 29 (23\%) out of 127 assessable vessels were determined as ischemic by CT. A total of 20 vessels (16\%) were determined as ischemic by SPECT. The inter-observer agreement was good $(\kappa=0.67)$. 
The AUC of the ROC curve of EEL was significantly higher than that of SPECT to predict ischemia-causing stenosis ( 0.86 ( $95 \%$ confidence interval (CI), $0.78-0.95)$ vs 0.67 (95\% CI, $0.57-0.77), p<0.005$ ) (Figure $2(a)$ ). The sensitivity ( $81 \%$ vs $42 \%, p=0.009$ ) and negative predictive value ( $95 \%$ vs $86 \%, p=0.04$ ) of EEL was significantly higher than SPECT (Figure 3 ), while the specificity $(92 \%$ vs $91 \%, p=1.0)$ and positive predictive value ( $72 \%$ vs $55 \%, p=0.24)$ did not show significant difference (Table 2). The diagnostic accuracy of EEL was significantly higher than SPECT to diagnose ischemia-causing stenosis (90\% vs $81 \%, p=0.04)$. The accuracy of EEL and SPECT was similar for the left anterior descending ( $74 \%$ vs $72 \%, p=0.81$ ), while it was marginally higher in the right coronary artery $(98 \%$ vs $85 \%, p=0.06)$ and significantly higher in the left circumflex artery ( $98 \%$ vs $86 \%, p=0.03$ ) (Table 2 ).

Table 2. Per-vessel diagnostic performance of EEL and SPECT to detect significant stenosis by FFR.

\begin{tabular}{|c|c|c|c|}
\hline & EEL & SPECT & $P$ \\
\hline \multicolumn{4}{|l|}{ All vessels } \\
\hline Accuracy & $90(83-94)$ & $81(73-88)$ & $0.04^{*}$ \\
\hline Sensitivity & $81(61-93)$ & $42(23-63)$ & $0.009^{*}$ \\
\hline Specificity & $92(85-97)$ & $91(84-96)$ & 1.0 \\
\hline Positive predictive value & $72(53-87)$ & $55(32-77)$ & 0.24 \\
\hline Negative predictive value & $95(89-98)$ & $86(78-92)$ & $0.04^{\star}$ \\
\hline \multicolumn{4}{|l|}{ Right coronary artery } \\
\hline Accuracy & $98(87-100)$ & $85(71-94)$ & 0.06 \\
\hline Sensitivity & $100(19-100)$ & $67(9-99)$ & 1.0 \\
\hline Specificity & $97(86-100)$ & $87(72-96)$ & 0.20 \\
\hline Positive predictive value & $75(19-99)$ & $29(4-71)$ & 0.24 \\
\hline Negative predictive value & $100(86-100)$ & $97(85-100)$ & 0.48 \\
\hline \multicolumn{4}{|l|}{ Left anterior descending } \\
\hline Accuracy & $74(59-87)$ & $72(56-85)$ & 0.81 \\
\hline Sensitivity & $71(42-92)$ & $36(13-65)$ & 0.13 \\
\hline Specificity & $76(57-90)$ & $90(73-98)$ & 0.30 \\
\hline Positive predictive value & $59(33-82)$ & $63(25-92)$ & 1.0 \\
\hline Negative predictive value & $85(65-96)$ & $74(57-88)$ & 0.37 \\
\hline \multicolumn{4}{|l|}{ Left circumflex } \\
\hline Accuracy & $98(88-100)$ & $86(72-95)$ & $0.03^{*}$ \\
\hline Sensitivity & $89(52-100)$ & $44(14-79)$ & 0.13 \\
\hline Specificity & $100(85-100)$ & $97(85-100)$ & 1.0 \\
\hline Positive predictive value & $100(52-100)$ & $80(28-99)$ & 0.38 \\
\hline Negative predictive value & $97(85-100)$ & $87(72-96)$ & 0.20 \\
\hline
\end{tabular}

Numbers in parenthesis represent $95 \%$ confidence interval. * Statistically significant, $p<0.05$. EEL, estimated energy loss; LAD, left anterior descending; LCX, left circumflex; FFR, fractional flow reserve; RCA, right coronary artery; SPECT, single photon emission computed tomography. 


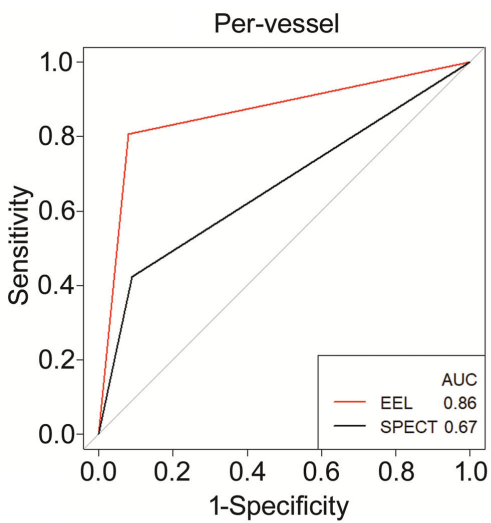

(a)

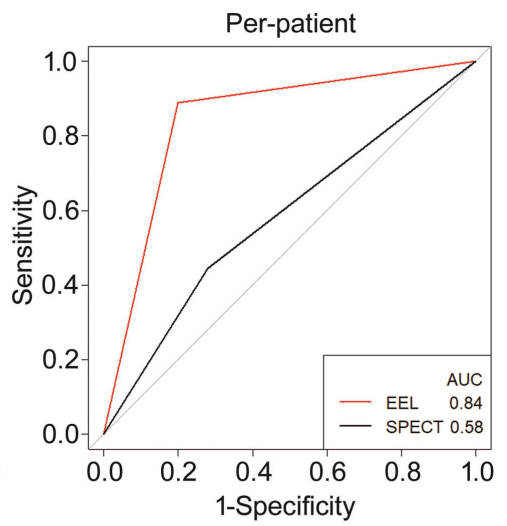

(b)

Figure 2. Receiver operating characteristics curves to predict ischemia-causing stenosis by fractional flow reserve on a per-vessel (a) and a per-patient (b) basis. The area under the curve of EEL was significantly higher than SPECT $(p<0.005)$. EEL, estimated energy loss; SPECT, single photon emission computed tomography.

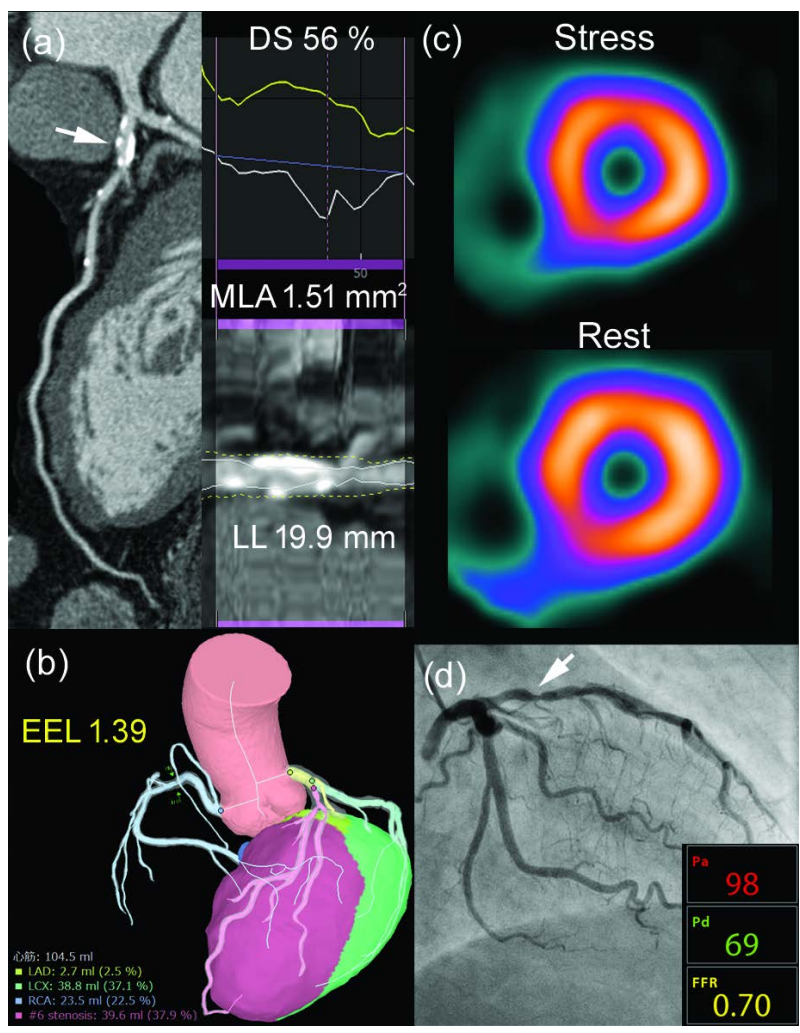

Figure 3. Curved planar reconstruction image and stenosis analysis performed by the software of a 70-year-old male suspected of angina (a); Calcified plaque at the proximal left anterior descending (a, arrow) showed 56\% stenosis with an MLA of $1.51 \mathrm{~mm}^{2}$ and LL of $19.9 \mathrm{~mm}$. The myocardial volume of the stenosis-related territory was $39.6 \mathrm{ml}$ (b); EEL was calculated as 1.39 , above the threshold for ischemia (>1.17); SPECT showed no stress-induced ischemia (c); Intermediate stenosis was present at proximal left anterior descending artery on angiography (d,arrow) and the FFR was 0.70 , positive for ischemia $(\leq 0.80)$ (d). DS, diameter stenosis; EEL, estimated energy loss; FFR, fractional flow reserve; LL, lesion length; MLA, minimal lumen area; SPECT, single photon emission computed tomography. 
There were 5 false-negatives for EEL (Figure 4) with an average value of 0.93 . CT underestimated the stenosis severity in 1 vessel and the average FFR of the remaining 4 vessels were 0.77 (range, 0.71 - 0.80). Conversely, EEL resulted as false-positive in 8 vessels and the average FFR was 0.86 (range, $0.82-0.90$ ). The average MLA and EEL were $1.04 \mathrm{~mm}^{2}$ (range, $0.73 \mathrm{~mm}^{2}-1.76 \mathrm{~mm}^{2}$ ) and 1.47 (range, $1.21-2.02$ ), respectively.

\subsection{Per-Patient Analysis}

A total of 21 patients were determined to have ischemia by EEL while 15 patients had ischemia by SPECT. The AUC of the ROC curve significantly improved from 0.58 (95\% CI, $0.43-0.73)$ to 0.84 (95\% CI, $0.74-0.95)(p<0.005$, Figure $2(\mathrm{~b})$ ). The sensitivity ( $89 \%$ vs $44 \%, p=0.01$ ) and negative predictive value $(91 \%$ vs $64 \%, p=0.04$ ) of EEL was higher than SPECT (Table 3 ). The diagnostic accuracy of EEL was significantly higher than SPECT to diagnose ischemic patients ( $84 \%$ vs $61 \%, p=0.01$ ).

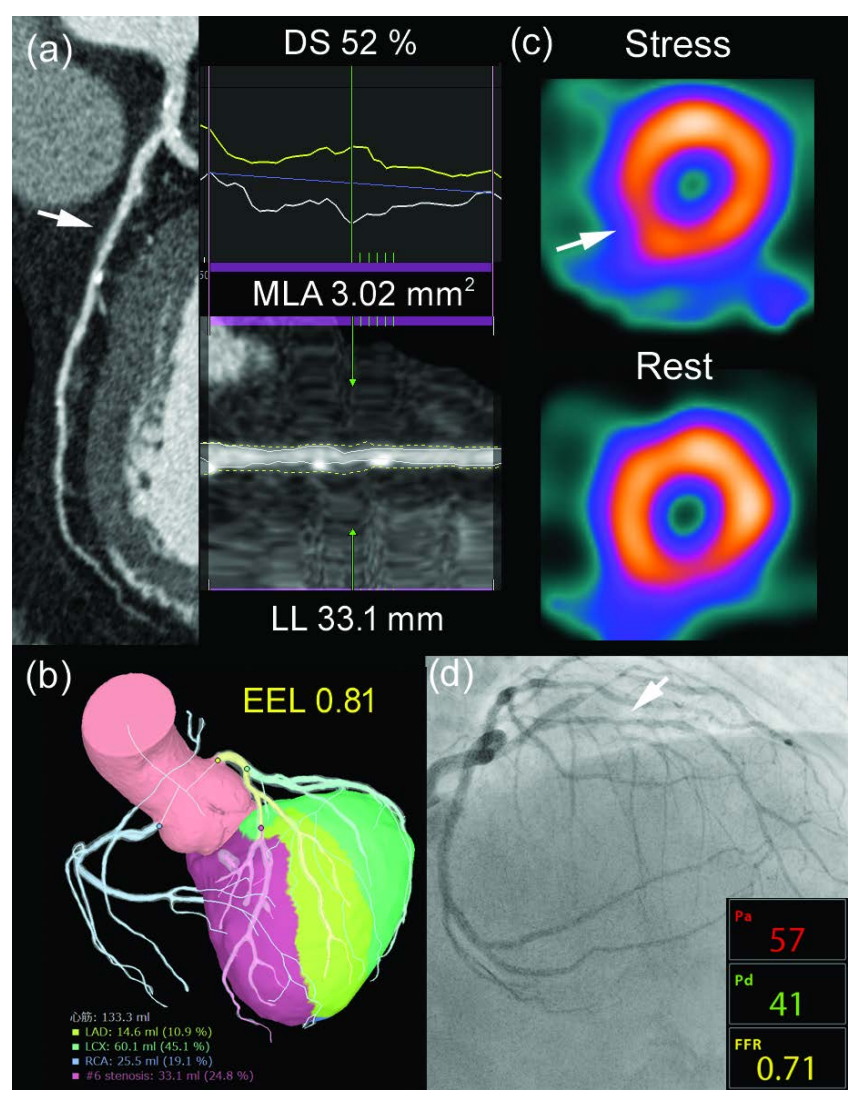

Figure 4. Curved planar reconstruction image and stenosis analysis performed by the software of an 81-year old male with chest discomfort (a); Diffuse plaque at the left anterior descending showed $52 \%$ stenosis with an MLA of $3.02 \mathrm{~mm}^{2}$ and LL of $33.1 \mathrm{~mm}$. The myocardial volume of the stenosis-related territory was $33.1 \mathrm{ml}(\mathrm{b})$; EEL was calculated as 0.81 , below the threshold for ischemia $(>1.17)$. SPECT showed stress-induced ischemia at the septum (c); FFR of 0.71 was positive for ischemia $(\leq 0.80)$ (d). DS, diameter stenosis; EEL, estimated energy loss; FFR, fractional flow reserve; LL, lesion length; MLA, minimal lumen area; SPECT, single photon emission computed tomography. 
Table 3. Per-patient diagnostic performance of EEL and SPECT to detect significant stenosis by FFR.

\begin{tabular}{cccc}
\hline & EEL & SPECT & $P$ \\
\hline Accuracy & $84(69-93)$ & $61(44-75)$ & $0.01^{*}$ \\
Sensitivity & $89(65-99)$ & $44(22-69)$ & $0.01^{*}$ \\
Specificity & $80(59-93)$ & $72(51-88)$ & 0.74 \\
Positive predictive value & $76(53-92)$ & $53(27-79)$ & 0.18 \\
Negative predictive value & $91(71-99)$ & $64(44-81)$ & $0.04^{*}$ \\
\hline
\end{tabular}

Numbers in parenthesis represent $95 \%$ confidence interval. EEL, estimated energy loss; FFR, fractional flow reserve; SPECT, single photon emission computed tomography. ${ }^{\star}$ Statistically significant, $p<0.05$.

\subsection{Discrepancy between EEL and SPECT}

There were 2 vessels which SPECT correctly detected ischemia but missed by EEL. The lesion length was very short $(3.2 \mathrm{~mm})$ in one lesion and the minimal lumen area was large $\left(3.02 \mathrm{~mm}^{2}\right)$ (Figure 4$)$, in the other lesion. Conversely, EEL overdiagnosed 7 vessels which SPECT correctly ruled out ischemia. The minimal lumen area was small $\left(<1 \mathrm{~mm}^{2}\right)$ or either lesion length was long $(>15 \mathrm{~mm})$.

SPECT misdiagnosed 11 vessels as non-ischemic which were ischemic by EEL and FFR. Five of these vessels were from multiple-vessel disease patients. The average FFR of the remaining vessels was 0.77 (range, $0.70-0.80$ ). Overdiagnosis of SPECT occurred in 8 vessels which were actually non-ischemic by FFR and correctly diagnosed by EEL. Five of these vessels were in the RCA territory, followed by LAD (2 vessels) and LCX (1 vessel).

\section{Discussions}

The present study showed that EEL improved the diagnostic accuracy from $81 \%$ to $90 \%$ to detect ischemia-causing stenosis by FFR compared with SPECT. This was achieved by increasing the sensitivity from $42 \%$ to $81 \%$ while preserving the high specificity of over $90 \%$. The major strengths of EEL are that supercomputer is unnecessary due to its simple calculation and that ischemia analysis could be performed without additional stress agent or radionuclide.

A meta-analysis investigating the diagnostic performance of SPECT to detect ischemia-causing stenosis by FFR showed that in spite of the high specificity of $84 \%$, the sensitivity was as low as $61 \%$ [10]. The low sensitivity of SPECT to detect FFR-positive stenosis was shown in other studies which ranged from 35\% to $68 \%$ [11] [12] [13]. The sensitivity of SPECT in the present study was $42 \%$ which was similar to the previous reports. The large spatial resolution of SPECT of about $10 \mathrm{~mm}$ might lower the sensitivity because it could be too large to detect small sub-endocardial ischemia. In addition, patients with extremely severe stenosis were not included in this study because additional test to prove ischemia is clinically unnecessary. Therefore, the proportion of moderate stenosis was high which might have decreased the sensitivity. This was in line with the Dan-NICAD study which showed a sensitivity of $36 \%$ using SPECT in a popula- 
tion of coronary artery disease suspected by coronary CT [14]. EEL would be superior to SPECT to diagnose ischemia in vessels with moderate stenosis.

The diagnosis of ischemia at the inferior wall is a challenge using SPECT because the diaphragm occasionally causes attenuation artifacts [13] [15]. This might have caused the low sensitivity of SPECT to detect ischemia at the right coronary artery in the present study. Prone imaging [13] [15] or CT attenuation correction [13] [16] could be used to improve the diagnostic accuracy of the inferior wall. Also, Cadmium-Zinc-Telluride detector SPECT would be superior to the conventional anger SPECT because of its higher count sensitivity [17]. The present study showed that EEL would be superior to SPECT to diagnose ischemia, especially at the inferior wall.

FFR $_{\mathrm{CT}}$ (HeartFlow; Redwood, CA) is a widely used method to predict ischemia-causing stenosis using coronary CT angiography data. It is calculated by a supercomputer using a sophisticated computed-flow dynamics model. A recent NXT trial showed that the sensitivity, specificity, and accuracy of $\mathrm{FFR}_{\mathrm{CT}}$ to detect ischemia by invasive FFR were $84 \%, 86 \%$, and $86 \%$, respectively [2]. CT-FFR (Canon Medical Systems; Tochigi, Japan) is another approach to estimate ischemia-causing stenosis using coronary CT angiography data. A study using CT-FFR showed that the sensitivity, specificity, and accuracy of CT-FFR to predict ischemia by FFR were $78 \%, 87 \%$, and $84 \%$, respectively. Although we did not compare the diagnostic accuracy of EEL with these methods, the diagnostic performance of EEL was similar even though we used a very simple calculation.

We acknowledge the following limitations. Since this was a retrospective study, selection bias might exist. Secondly, this was a single-center study with limited amount of cohort and needs to be validated by a large multi-center study. Thirdly, the coronary flow at maximal hyperemia was estimated at the same value in all patients. Because the microvascular function differs in each patient, this estimation might not be true.

\section{Conclusion}

In conclusion, EEL diagnosed ischemia-causing stenosis proven by invasive FFR better than SPECT by improving the sensitivity. Because EEL could be calculated easily using the coronary CT data, this would help clinicians to diagnose ischemic lesions on site without additional medication.

\section{Funding}

This study was supported by Bayer research grant 2018 of the Japanese Radiological Society.

\section{Conflicts of Interest}

The authors declare no conflicts of interest regarding the publication of this paper. 


\section{References}

[1] Tonino, P., De Bruyne, B., Pijls, N., et al. (2009) Fractional Flow Reserve versus Angiography for Guiding Percutaneous Coronary Intervention. The New England Journal of Medicine, 360, 213-224. https://doi.org/10.1056/NEJMoa0807611

[2] Nørgaard, B.L., Leipsic, J., Gaur, S., et al. (2014) Diagnostic Performance of Noninvasive Fractional Flow Reserve Derived from Coronary Computed Tomography Angiography in Suspected Coronary Artery Disease: The NXT Trial (Analysis of Coronary Blood Flow Using CT Angiography: Next Steps). Journal of the American College of Cardiology, 63, 1145-1155. https://doi.org/10.1016/j.jacc.2013.11.043

[3] Tomizawa, N., Yamamoto, K., Inoh, S., Nojo, T. and Nakamura, S. (2018) Simplified Bernoulli Formula to Predict Flow Limiting Stenosis at Coronary Computed Tomography Angiography. Clinical Imaging, 51, 104-110. https://doi.org/10.1016/j.clinimag.2018.01.018

[4] Tomizawa, N., Yamamoto, K., Hayakawa, Y., et al. (2017) A Clinical Model to Identify Patients with High-Risk Plaque by Coronary Computed Tomography Angiography. International Journal of Cardiology, 228, 260-264. https://doi.org/10.1016/j.ijcard.2016.11.248

[5] Tomizawa, N., Hayakawa, Y., Inoh, S., Nojo, T. and Nakamura, S. (2015) Clinical Utility of Landiolol for Use in Coronary CT Angiography. Research Reports in Clinical Cardiology, 6,145-152. https://doi.org/10.2147/RRCC.S77559

[6] Agatston, A., Janowitz, W., Hildner, F., et al. (1990) Quantification of Coronary Artery Calcium Using Ultrafast Computed Tomography. Journal of the American College of Cardiology, 15, 827-832. https://doi.org/10.1016/0735-1097(90)90282-T

[7] Zipes, D., Libby, P., Bonow, R. and Braunwald, E. (2004) Heart Disease: A Textbook of Cardiovascular Medicine. 7th Edition, Elsevier Science, Philadelphia, PA.

[8] Cohen, J. (1960) A Coefficient of Agreement for Nominal Scales. Educational and Psychological Measurement, 20, 37-46. https://doi.org/10.1177/001316446002000104

[9] De Long, E.R., De Long, D.M. and Clarke-Pearson, D.L. (1988) Comparing the Areas under Two or More Correlated Receiver Operating Characteristic Curves: A Nonparametric Approach. Biometrics, 44, 837-845. https://doi.org/10.2307/2531595

[10] Takx, R.A.P., Blomberg, B.A., Aidi, H.E., et al. (2015) Diagnostic Accuracy of Stress Myocardial Perfusion Imaging Compared to Invasive Coronary Angiography with Fractional Flow Reserve Meta-Analysis. Circulation: Cardiovascular Imaging, 8, e002666-e002666. https://doi.org/10.1161/CIRCIMAGING.114.002666

[11] Neglia, D., Rovai, D., Caselli, C., et al. (2015) Detection of Significant Coronary Artery Disease by Noninvasive Anatomical and Functional Imaging. Circulation: Cardiovascular Imaging, 8, 1-10.

[12] Chung, H.W., Ko, S.M., Hwang, H.K., et al. (2017) Diagnostic Performance of Coronary CT Angiography, Stress Dual-Energy CT Perfusion, and Stress Perfusion Single-Photon Emission Computed Tomography for Coronary Artery Disease: Comparison with Combined Invasive Coronary Angiography and Stress Perfusion Cardiac MRI. Korean Journal of Radiology, 18, 476-486. https://doi.org/10.3348/kjr.2017.18.3.476

[13] Ito, S., Endo, A., Okada, T., et al. (2017) Comparison of CTAC and Prone Imaging for the Detection of Coronary Artery Disease Using CZT SPECT. Annals of Nuclear Medicine, 31, 629-635. https://doi.org/10.1007/s12149-017-1194-z

[14] Nissen, L., Winther, S., Westra, J., et al. (2018) Diagnosing Coronary Artery Disease 
after a Positive Coronary Computed Tomography Angiography: The Dan-NICAD Open Label, Parallel, Head to Head, Randomized Controlled Diagnostic Accuracy Trial of Cardiovascular Magnetic Resonance and Myocardial Perfusion Scintigraphy. European Heart Journal-Cardiovascular Imaging, 19, 369-377. https://doi.org/10.1093/ehjci/jex342

[15] Nakaya, K., Onoguchi, M., Nishimura, Y., et al. (2017) Criteria for the Addition of Prone Imaging to Myocardial Perfusion Single-Photon Emission Computed Tomography for Inferior Wall. Nuclear Medicine Communications, 38, 748-755. https://doi.org/10.1097/MNM.0000000000000713

[16] Huang, J.-Y., Yen, R.-F., Lee, W.-C., et al. (2018) Improved Diagnostic Accuracy of Thallium-201 Myocardial Perfusion Single-Photon Emission Computed Tomography with CT Attenuation Correction. Journal of Nuclear Cardiology, 1-12. https://doi.org/10.1007/s12350-018-1230-y

[17] Niimi, T., Nanasato, M., Sugimoto, M. and Maeda, H. (2017) Evaluation of Cadmium-Zinc-Telluride Detector-Based Single-Photon Emission Computed Tomography for Nuclear Cardiology: A Comparison with Conventional Anger Single-Photon Emission Computed Tomography. Nuclear Medicine and Molecular Imaging, 51, 331-337. https://doi.org/10.1007/s13139-017-0474-9 